\title{
A Coupled Plasma and Sheath Model for High Density Reactors
}

\author{
Deepak Bose*, T.R. Govindan, and M. Meyyappan
}

NASA Ames Research Center, Moffett Field, CA 94035

\begin{abstract}
We present a coupled plasma and collisionless sheath model for the simulation of high density plasma processing reactors. Due to inefficiencies in numerical schemes and the resulting computational burden, a coupled multidimensional plasma and sheath simulation has not been possible model for gas mixtures and high density reactors of practical interest. In this work we demonstrate that with a fully implicit algorithm and a refined computational mesh, a self-consistent plasma and sheath simulation is feasible. We discuss the details of the model equations, the importance of ion inertia, and the resulting sheath profiles for argon and chlorine plasmas. We find that at low operating pressures (10-30 mTorr), the charge separation occurs only within a $0.5 \mathrm{~mm}$ layer near the surface in a 300 $\mathrm{mm}$ inductively coupled plasma etch reactor. A unified model eliminates the use of off-line or loosely coupled sheath models with simplifying assumptions which generally lead to uncertainties in ion flux and sheath electrical properties.
\end{abstract}

* Eloret Corp. 